\title{
On the nature of rate-dependent processing during phonetic perception
}

\author{
JOANNE L. MILLER \\ Northeastern University, Boston, Massachusetts \\ IONA L. AIBEL \\ Brown University, Providence, Rhode Island \\ and \\ KERRY GREEN \\ Northeastern University, Boston, Massachusetts
}

\begin{abstract}
We previously reported (Miller \& Liberman, 1979) that when listeners use transition duration to identify syllables as $/ \mathrm{ba} /$ or $/ \mathrm{wa} /$, they do so in relation to the overall duration of the syllables: As the syllables become longer and are perceived as having been produced at a slower rate of speech, the transition duration that differentiates $/ \mathrm{ba} /$ from $/ \mathrm{wa} /$ also becomes longer. In the present experiments, we investigated whether the adjustment for rate was based on the actual physical rate of the syllables (i.e., syllable duration) or on their subjective rate. We used an anchoring procedure to alter the subjective rate of the syllables while keeping physical rate constant, and then assessed the effect of this manipulation on the identification of the syllables as $\mathrm{ha} /$ or $/ \mathrm{wa} /$. We found that changes in subjective rate induced by the anchoring procedure had no reliable effect on syllable identification. However, in a control study, changes in physical rate that produced equivalent changes in subjective rate did reliably alter identification of the syllables. These findings indicate that, during phonetic perception, listeners accommodate for changes in the physical rate of speech, not for changes in its subjective rate.
\end{abstract}

Many of the acoustic properties that specify the identity of phonetic segments are temporal in nature. For example, a change in voice onset time can signal a change in voicing, as in "bath" versus "path", a change in the duration of the relatively steady-state vocalic portion of a word can signal a change in vowel quality, as in "bed" versus "bad," and a change in the duration of the initial formant transitions can signal a change in manner of articulation, as in "bill" versus "will." For temporal properties of this type, a relatively short duration of the critical

This research was supported by NINCDS Grant NS 14394, NINCDS RCDA NS 00661, NICHHD Contrast NIH 71-2420, and HEW BRSG RR 07143. We thank A. M. Liberman for making available the facilities of Haskins Laboratories and S. E. Blumstein and P. Lieberman for making available the facilities of the Phonetics Laboratory at Brown University for the preparation of stimulus materials. We also thank P. D. Eimas for valuable discussions throughout the course of the project and for comments on an earlier version of the manuscript. The data reported in this paper were first presented at the spring meeting of the Acoustical Society of America, Cincinnati, May 1983. Experiments 1 and 2 are the basis of an Honors Thesis by the second author, directed by the first author, submitted for the $\mathrm{ScB}$ degree at Brown University. Iona L. Aibel is now at New York University. Requests for reprints should be addressed to Joanne L. Miller, Department of Psychology, 282 NI, Northeatern University, Boston, Massachusetts 02115. acoustic property specifies one phonetic segment, whereas a longer duration specifies another.

In natural speech, however, the actual durations of temporal properties such as these are not constant, but vary systematically, and considerably, as a function of a number of factors, among them stress pattern, intonation contour, lexical and syntactic structure, and discourse structure (see Klatt, 1976, for a review). Yet another factor which introduces durational variation, and which is the focus of the present research, is speaking rate. It has been known for some time that speakers do not maintain a constant overall rate of speaking when they engage in conversation. And, although most of the change in overall rate is due to a change in the number and duration of pauses in the utterance, there is also modification in the rate at which the actual speech is produced-that is, in the rate of articulation (Goldman-Eisler, 1968; Grosjean \& Deschamps, 1975). Moveover, recent evidence has indicated that, for a given speaker, this change in rate of articulation can be substantial (Miller, Grosjean, Gee, \& Lomanto, Note 1). Specifically, speakers in the Miller et al. study often effected more than a threefold change in articulation rate when responding to a question in an interview situation, such that the average duration of syllables could range from less than 
$150 \mathrm{msec}$ to more than $450 \mathrm{msec}$. From the perspective of phonetic perception, the important question is whether such changes in syllable duration involve changes in the duration of those acoustic properties for which duration specifies phonetic identity. The answer would appear to be yes. In a number of experiments in which speech was elicited from speakers at different rates, it has been found that the durations of phonetically relevant temporal properties systematically change as overall rate changes (e.g., Miller \& Baer, 1983; Port, 1981; Summerfield, Note 2).

Given that a change in speaking rate can alter the durational characteristics of these linguistically relevant properties, the issue that immediately arises is how the listener is able to use them to specify a constant phonetic segment. One way to maintain perceptual constancy across rate variation is to treat the duration of these properties not in an absolute fashion, but in relation to the rate at which they were produced. And, indeed, there is considerable evidence that listeners do just this. Most often, this relational processing has been seen as a shift in the criterion duration of the property that distinguishes the two phonetic segments in question; as speech is slowed, the criterion duration of the property becomes longer. A review of the literature indicates that this type of processing exists for a host of temporal properties, specifying many phonetic contrasts (Miller, 1981a).

The purpose of the present research was to explore further the listener's perceptual adjustment for rate during phonetic perception. Our specific question concerned the nature of the rate information to which the listener adjusts. Two alternatives can be distinguished. The first, which we will term the subjective rate hypothesis, is that the listener's adjustment is mediated by the subjective rate of speech. As the speaker produces changes in rate of speech, the listener perceives the speech to be faster or slower and, on the basis of these changes in perceived rate, adjusts the way in which durational properties are used to specify phonetic identity. The second alternative, which we will designate the physical rate hypothesis, is that the adjustment does not involve reference to the subjective rate of speech at all. Rather, the adjustment is made directly to the variation in the physical rate of speech; the changes in subjective rate that typically accompany this variation play no causal role.

To help clarify the two alternatives, let us consider an experiment by Miller and Liberman (1979), which forms the basis for the current research. They focused on the distinction between syllable-initial stop consonant and semivowel in the syllables $/ \mathrm{ba} /$ and /wa/. For their experiment, they created a set of speech continua. Each continuum varied in the duration of the initial formant transitions; syllables with short transitions were heard as / $\mathrm{ba} /$ and those with longer transitions were heard as $/ \mathrm{wa} /$. The continua differed from each other in the overall duration of the syllables, which produced a change in subjective speaking rate-longer syllables were perceived as being articulated more slowly. The primary finding of the study was that the criterion duration value of the initial formant transitions for distinguishing / ba/ from /wa/ varied systematically as a function of syllable duration. As the syllables became longer, a longer transition duration was required to perceive $/ w a /$, as opposed to $/ \mathrm{ba} /$. The authors interpreted these findings as indicating that listeners were adjusting for a change in speaking rate, specified in this case by a change in overall syllable duration.

Consider how these results are to be explained in terms of the two views of rate adjustment outlined above. According to the subjective rate hypothesis, the change in syllable duration across the series altered the subjective rate of the syllables and the listener adjusted for this change in subjective rate when identifying the syllables as /ba/ or /wa/ on the basis of transition duration. According to the physical rate hypothesis, the change in subjective rate that accompanied the change in syllable duration was not causal. Rather, the listener adjusted for the change in overall syllable duration, which is directly related to the physical rate of the speech. Clearly, since in the Miller and Liberman experiment subjective rate and physical rate were confounded, both accounts of rate adjustment can readily accommodate the results.

The purpose of the present research was to dissociate physical and subjective rate in order to determine which of these variables influences phonetic perception. Specifically, we used an anchoring procedure to induce changes in subjective rate, while keeping physical rate (syllable duration) constant, and then measured the effect of this manipulation on the location of the /ba-wa/ category boundary. If the subjective rate hypothesis is correct, the change in subjective rate should lead to a modification in the /ba-wa/ category boundary, whereas if the physical rate hypothesis is correct, it should not.

\section{EXPERIMENT 1}

In preparation for the main experiment, we undertook a preliminary study that was designed to serve two purposes. The first was to replicate Miller and Liberman's (1979, Experiment 1) finding that, within certain limits, the transition duration at the /ba-wa/ category boundary increases as a function of increasing syllable duration. The second was to establish that, as informal listening tests suggested, changes in overall syllable duration produce systematic alterations in the subjective rate of the syllables. 


\section{Method}

Subjects. The subjects were 10 volunteer college students who were paid for their participation in the experiment. None reported any history of a speech or hearing disorder.

Stimuli. The stimuli consisted of seven /ba-wa/ series that differed from each other in overall syllable duration, and were created as follows. One basic three-formant /ba-wa/ series was generated on the Haskins Laboratories parallel resonance synthesizer, using the parameter values reported in Miller and Liberman (1979). All syllables within this series were $296 \mathrm{msec}$ in duration. Each consisted of a fixed 16-msec period of prevoicing (first formant only), ${ }^{1}$ a variable duration of formant transition, and a variable duration of steady-state vowel. The duration of the formant-transition segment increased across the series from 16 to $64 \mathrm{msec}$, in four msec steps. In order to maintain a constant syllable duration $(296 \mathrm{msec})$ across the series, as the transition was made longer, the steady-state vowel segment was shortened by an equivalent amount. For all stimuli, the first formant began at $234 \mathrm{~Hz}$ and rose in a stepwise linear fashion to its steady-state value of $769 \mathrm{~Hz}$, the second formant began at $616 \mathrm{~Hz}$ and rose in a stepwise linear fashion to its steady-state value of $1232 \mathrm{~Hz}$, and the third formant remained constant at $2862 \mathrm{~Hz}$. The overall amplitude of each syllable increased by $28 \mathrm{~dB}$ over the course of the prevoicing and transition segment and remained constant thereafter, and the fundamental frequency was kept constant at $114 \mathrm{~Hz}$ throughout the syllable. ${ }^{2}$

The 13 stimuli in this /ba-wa/ series were recorded on audio tape, subsequently digitized at a $10-\mathrm{kHz}$ sampling rate on a PDP. 11/34 computer, and edited as follows. For each stimulus, six additional versions were created by deleting successively larger seg. ments of steady-state vowel from the end of the syllable, in 36msec chunks. This manipulation yielded a total of seven versions of each stimulus, having overall syllable durations of $80,116,152$, $188,224,260$, and $296 \mathrm{msec}$, and hence seven /ba-wa/ series differing from each other only in syllable duration, in particular, in the extent of the steady-state vowel segment.

Ten different randomized test orders were constructed with these stimuli. Each order contained one instance of each of the 91 syllables ( 7 series $\times 13$ syllables each). The syllables were recorded on audio tape with an onset-to-onset interval of $2.9 \mathrm{sec}$.

Procedure. Each subject participated in five sessions, conducted on different days. The first session was considered a pretest and the data were not included in the final analyses. The purpose of the pretest was twofold: first, to familiarize the listeners with the set of stimuli (this was particularly important for the rate-judgment task) and, second, to ensure that each listener could consistently identify the syllables as $/ b a /$ or $/ w a /$. No subjects were excluded from the first experiment on the basis of pretest data.

In each of the five sessions, the subjects performed two tasks, syllable identification and rate judgment. For the syllable-identification task, the listener was instructed to identify each syllable as /ba/ or /wa/ by writing B or W on an answer sheet. The subject was told to respond on every trial, guessing if the stimulus was ambiguous. For the rate-judgment task, the subject was instructed to judge the speaking rate at which each syllable was produced by circling a number from 1 to 10 on a scale. Low numbers specified a slow speaking rate and high numbers specified a fast speaking rate. The subject was told to judge the rate of each syllable in relation to the range of syllable rates encountered in the experiment, rather than the range of rates encountered in natural conversation. Again, subjects were told to be sure to respond on every trial.

During the pretest session, 6 of the 10 test orders were presented to the subjects, in the following order: 6-8, 1-3. During each of the subsequent four sessions, all 10 test orders were presented, as follows: Sessions 2 and 4, orders 1-5, 6-10; Sessions 3 and 5, orders 610, 1-5. Five of the subjects always performed the syllableidentification task during the first half of the session and the ratejudgment task during the second half; the other five subjects performed these tasks in the converse order. Across Sessions 2 through 5 , each subject provided 20 identification responses and 20 rate judgments for each stimulus.
The stimuli were presented binaurally to subjects through matched TDH-39 earphones. The level controls were set such that the peak vowel level for the endpoint /ba/ (16-msec transition duration) from the $296-\mathrm{msec}$ series was $78 \mathrm{~dB}$ SPL. With this calibration procedure, any inherent variation in amplitude across the different syllables was maintained throughout the experiment.

\section{Results and Discussion}

A first question to consider is whether we were successful in replicating Miller and Liberman's (1979) finding. For each subject, the percentage of /ba/ responses was calculated for each stimulus of the seven /ba-wa/ series, based on 20 responses per item. The mean identification functions, pooled across the 10 listeners, are shown in Figure 1. It is apparent that the listeners' responses were orderly: Syllables with relatively short transitions were consistently labeled $/ \mathrm{ba} /$, and those with longer transitions were consistently labeled /wa/. But more important for our purposes, the transition duration at which the responses changed from predominantly $/ \mathrm{ba} /$ to predominantly /wa/ was not constant; rather, it increased systematically with increasing syllable duration.

In order to obtain a summary measure of the shift in identification function with increasing syllable duration, we calculated for each series, for each subject, the location of the /ba-wa/ category boundary. This was done by fitting a linear regression line to the data in the boundary region of the identification curve, and taking as the boundary value that score which corresponded to $50 \% / \mathrm{ba} /$ responses. The mean boundary values for each series (in millisecond transition duration), averaged across the 10 listeners, were as follows: $30.83(80), 36.94$ (116), 41.05 (152), 43.36 (188), 43.61 (224), 44.87 (260), 46.99 (296). Thus, as would be expected on the basis of the identification functions, the progression of mean boundary values is orderly. Note in addition that the magnitude of shift in boundary location with a given

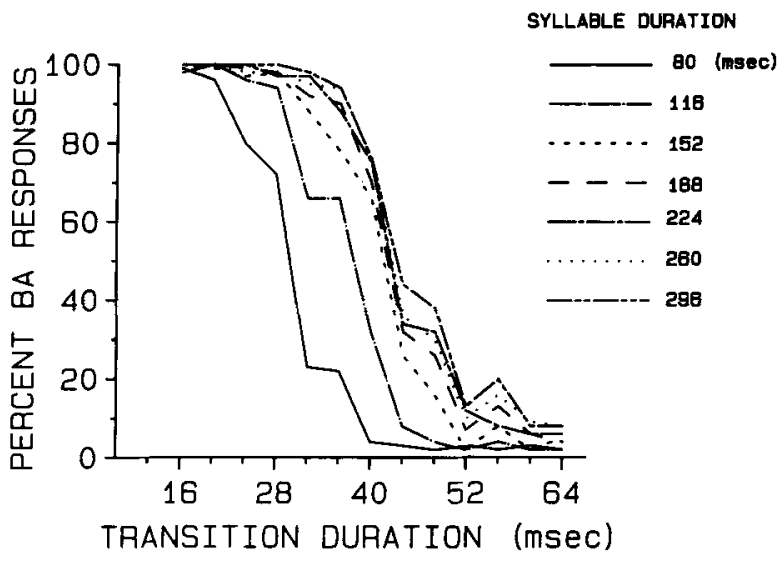

Figure 1. Percentage of /ba/ responses as a function of transltion duration for seven /ba-wa/ series varying in overall syllable duration from 80 to 296 msec. Experiment 1. 
increase in syllable duration is not constant, but decreases as the syllables become longer. This overall pattern of results and, indeed, the actual boundary values are in close agreement with the original findings of Miller and Liberman (1979).

In anticipation of Experiment 2, which employed stimuli from three of the series, namely, the 80-, 116-, and 152-msec series, it was important to establish that the boundary locations for these three series were reliably different from one another. To do this, we entered the individual boundary scores for all seven series into a one-way repeated measures analysis of variance. As expected, the overall effect of syllable duration was highly significant $[F(6,54)=$ $24.93, \mathrm{p}<.0011$. We then conducted individual comparisons on the three series of interest, ${ }^{3}$ which revealed that the boundary locations for the 80- and 116 -msec series were reliably different $(p<.01)$, as were those for the 116- and 152-msec series $(\mathrm{p}<.02)$.

Consider next the rate-judgment data. We calculated for each subject the mean rate judgment for each syllable, averaged over 20 trials. The group data, pooled over the 10 subjects, are displayed in Figure 2. The rate judgments of the subjects were also very orderly: As the overall syllable duration increased, the subjects assigned the syllables increasingly lower scores; that is to say, they judged them to be slower. The mean rate judgments for each series, pooled across all 13 stimuli, were as follows: $\mathbf{8 . 8 8}$ (80), 7.66 (116), 6.43 (152), 5.21 (188), 4.18 (224), 3.43 (260), 2.82 (296). Again, in anticipation of Experiment 2 , it was important to determine that the rate judgments for the 80-, 116-, and 152-msec series differed reliably. We conducted a one-way repeated measures analysis of variance on the individual mean rate judgments (pooled across the stimuli in each of the seven series), and obtained, as expected, a highly reliable effect of syllable duration $[F(6,54)=257.54$,

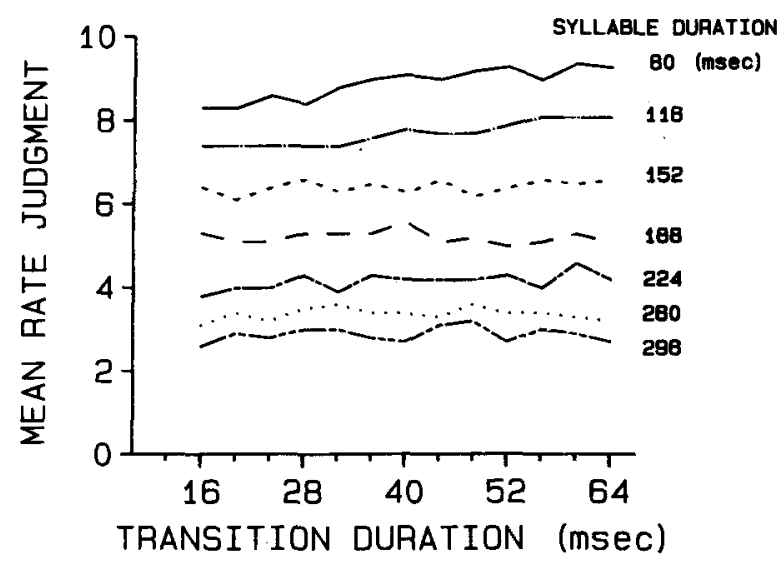

Figure 2. Mean rate judgment for syllables from seven /ba-wa/ series varying in overall syllable duration from 80 to 296 msec. Experiment 1. $\mathrm{p}<.001$ ]. Individual comparisons indicated that the rate judgments for both the 80 - and the $152-\mathrm{msec}$ series differed reliably from those for the $116-\mathrm{msec}$ series $(p<.01$ in each case).

The results of Experiment 1 corroborate Miller and Liberman's finding that when listeners use transition duration to identify syllables as $/ \mathrm{ba} /$ or $/ \mathrm{wa} /$, they do so in relation to the overall duration of the syllable. In addition, they establish that a modification in the duration of the syllables produces systematic changes in subjective rate. We are now able to address our main experimental question: Was it the change in physical rate or the change in subjective rate that was responsible for the shift in category boundary?

\section{EXPERIMENT 2}

In order to establish whether physical rate or subjective rate is the determining factor in phonetic perception, it is necessary to dissociate the two types of rate information and then assess which type is responsible for alterations in category boundary location. To separate the two types of rate information, we used a standard anchoring procedure (e.g., Sawusch \& Nusbaum, 1979; Simon \& StuddertKennedy, 1978; and see Helson, 1964; Parducci, 1975), whereby subjective rate could be altered while keeping physical rate constant. More specifically, we predicted that if syllables from a given /ba-wa/ series, called the target series, were presented in the context of numerous faster syllables, they would be perceived contrastively as relatively slow. Conversely, if the target syllables were presented in the context of many slower syllables, they would be perceived contrastively as relatively fast. If the critical rate information for phonetic perception is subjective rate, then the alteration in subjective rate induced by the anchoring procedure should lead to systematic shifts in the location of the category boundary on the target series. However, if it is physical rate that is critical, then the boundary location should not change, since physical rate has remained constant.

The stimuli were a subset of those used in Experiment 1 . The target series was the 116-msec series; specifically, the 12 stimuli from the series with transition durations ranging from 16 to $60 \mathrm{msec}{ }^{4}$ Our choice of target series was motivated primarily by the requirement to choose a series whose category boundary could, in principle, be shifted in either direction. As established by the analyses of Experiment 1 (and see Figure 1), the 116-msec series meets this constraint, since the /ba-wa/ category boundary can be located at a shorter transition duration (as is true for the 80-msec series) and it can be located at a longer transition duration (as is true for the $152-\mathrm{msec}$ series). Thus, a failure to find a shift in boundary 
location with changes in subjective rate induced by the anchoring procedure could not be attributed to floor or ceiling effects on boundary location.

To provide a context of fast syllables, we chose two clear exemplars from the 80-msec series, the $/ \mathrm{ba} /$ with a transition duration of $16 \mathrm{msec}$ and the /wa/ with a transition duration of $60 \mathrm{msec}$. The syllables we chose to provide a slow context were the $/ \mathrm{ba} /$ and /wa/ from the $152-\mathrm{msec}$ series with $16-$ and $60-\mathrm{msec}$ transition durations, respectively. Two comments about our choice of context, or anchoring, stimuli are in order. First, it was imperative that subjects perceive the rate of the anchoring syllables as different from the rate of the target syllables. As the ratejudgment data analyses of Experiment 1 had indicated (and see Figure 2), this was indeed the case with the 80-, 116-, and 152-msec stimuli. Second, note that the anchoring syllables included only good exemplars of $/ \mathrm{ba} /$ and $/ \mathrm{wa} /$ (those with transition durations of 16 and $60 \mathrm{msec}$, respectively) rather than members of the entire /ba-wa/ series. The reason for this is that, since the boundary location is different for the 80- and 152-msec series, the two series actually contain different numbers of $/ \mathrm{ba} / \mathrm{and} / \mathrm{wa} /$ tokens. Consequently, if we had used the entire 80and $152-\mathrm{msec}$ series as anchoring stimuli, we might have inadvertently induced selective adaptation effects-seen as boundary shifts-by exposing listeners to relatively many $/ \mathrm{ba} / \mathrm{s}$ in the case of the $152-\mathrm{msec}$ series or many /wa/s in the case of the 80-msec series (cf. Eimas \& Corbit, 1973). To ensure that any boundary shifts we might obtain on the 116-msec target series were, in fact, due to changes in subjective rate induced by exposure to the anchoring stimuli, and not due to selective adaptation effects, we used an equal number of clear $/ \mathrm{ba} / \mathrm{s}$ and $/ \mathrm{wa} / \mathrm{s}$ to provide the context.

The stimuli described above were used to construct test tapes for three conditions, a neutral anchoring condition, a fast anchoring condition, and a slow anchoring condition. All tapes contained instances of all the stimuli-the medium-rate target series (116msec duration), the fast anchoring syllables (80msec duration), and the slow anchoring syllables (152msec duration). The tapes differed only in the proportion of fast-to-slow anchoring syllables that they contained. All subjects were tested in all conditions. Their task, on each trial, was to indicate whether the syllable was $/ \mathrm{ba} /$ or $/ \mathrm{wa} /$ and to judge its rate. Detailed specifications of the stimulus materials and procedures are given below.

If the anchoring procedure is successful in inducing contrast effects, we will obtain the following results regarding the rate judgments for the $116-\mathrm{msec}$ target syllables. In the fast anchoring condition, the stimuli will be perceived as relatively slow and, hence, will receive relatively low rate judgments, compared to the neutral anchoring condition. In the slow anchoring condition, however, they will be perceived as relatively fast and, thus, will receive relatively high rate judgments, again compared with the neutral anchoring condition. As for the identification responses, the following predictions can be made. If phonetic identification depends on subjective rate, then the boundary locations for the 116-msec target series should be reliably different across the conditions. Specifically, the boundary value for the slow anchoring condition, where the syllables are perceived as relatively fast, should be lower than that for the neutral anchoring condition and, conversely, the boundary value for the fast anchoring condition, where the syllables are perceived as relatively slow, should be higher than that for the neutral anchoring condition. If, however, identification of $/ \mathrm{ba} /$ and /wa/ depends on physical rate, then the boundary location for the 116-msec target series should not differ reliably across the three conditions.

\section{Method}

Subjects. Fourteen college students served as paid volunteers in this experiment. None had participated in Experiment 1, and none reported any history of a speech or hearing disorder.

Stimuli. A subset of stimuli from Experiment 1, drawn from the $80-, 116-$, and 152-msec /ba-wa/ series, was used to create test tapes for the neutral, fast, and slow anchoring conditions. For each condition, 10 randomized test orders were created, each containing 36 stimuli, as specified below. All stimuli were recorded with a constant onset-to-onset interval of $4.4 \mathrm{sec}$.

For the neutral anchoring condition, each test order contained the following 36 stimuli: Six instances of the $16-\mathrm{msec} / \mathrm{ba} /$ and six instances of the $60-\mathrm{msec} / \mathrm{wa} /$ from the $80-\mathrm{msec}$ series, six instances of the $16-\mathrm{msec} / \mathrm{ba} /$ and six instances of the $60-\mathrm{msec} / \mathrm{wa} /$ from the 152-msec series, and one instance of each of the $12 \mathrm{stim}$ uli from the 116-msec series with transition durations ranging from 16 to $60 \mathrm{msec}$. Thus, there was an equal number of fast $(80 \mathrm{msec})$, medium (116 msec), and slow (152 msec) syllables.

For the fast anchoring condition, each test order contained the following 36 stimuli: Nine instances of the $16-\mathrm{msec} / \mathrm{ba} /$ and nine instances of the $60-\mathrm{msec} / \mathrm{wa} /$ from the 80 -msec series, three instances of the $16-\mathrm{msec} / \mathrm{ba} /$ and three instances of the $60-\mathrm{msec}$ /wa/ from the 152-msec series, and one instance of each of the 12 stimuli from the 116-msec series with transition durations ranging from 16 to $60 \mathrm{msec}$. Thus, the ratio of fast $/$ medium/slow syllables was $3 / 2 / 1$.

For the slow anchoring condition, each test order contained the following 36 stimuli: Three instances of the $16-\mathrm{msec} / \mathrm{ba} /$ and three instances of the $60-\mathrm{msec} / \mathrm{wa} /$ from the 80 -msec series, nine instances of the 16-msec / $\mathrm{ba} /$ and nine instances of the $60-\mathrm{msec}$ /wa/ from the 152-msec series, and one instance of each of the 12 stimuli from the 116-msec series with transition durations ranging from 16 to $60 \mathrm{msec}$. Thus, the ratio of fast/medium/slow syllables was $1 / 2 / 3$.

Procedure. Each of the 14 subjects participated in five sessions, including one pretest session and four test sessions. During the pretest session, the listeners were presented with the 10 orders for the neutral anchoring condition, 1-10. The pretest data were not included in the main analyses. However, one subject was excluded on the basis of the pretest data for failing to consistently identify the test syllables as /ba/ and /wa/, and was replaced by an additional subject. During each of the subsequent four test sessions, all listeners were presented first with five of the neutral anchoring condition test orders (Days 2 and 4, Orders 1-5; Days 3 and 5, Orders 6-10). They were then presented with all 10 orders (1-10) for either the fast anchoring condition or the slow anchoring 
condition: Half of the subjects were tested in the fast condition in Sessions 2 and 3 and in the slow condition in Sessions 4 and 5 , and the other half of the subjects were tested in the reverse order.

The subjects were instructed to make two judgments for each stimulus, on each trial. They were to first identify the syllable as $/ b a /$ or /wa/ by writing a B or W on an answer sheet next to the trial number, and then to judge the rate of the syllable by circling a number from 1 to 10 on a scale also located next to the trial number. As in the previous experiment, the subjects were informed that low numbers signified relatively slow speaking rates and high numbers signified relatively fast rates. In addition, the listeners were again requested to judge the rate of each syllable in relation to the range of rates encountered in the experiment, rather than the range of rates encountered in natural conversation. Note that from the subject's point of view there was no differentiation between target and anchoring syllables. Across the 4 days of testing (excluding the pretest), each subject provided 20 identification responses and 20 rate judgments for each of the 116-msec target syllables under each of the three anchoring conditions.

As in the first experiment, the stimuli were presented to subjects binaurally through matched TDH-39 earphones. And, again, the level controls were set such that the peak reading for the endpoint / ba/ (16-msec transition duration) of the 296-msec series was $78 \mathrm{~dB}$ SPL.

\section{Results and Discussion}

The first question to be addressed is whether the anchoring procedure was successful in generating a modification in subjective rate. To answer this question, we calculated, for each subject, the mean rate judgment for each of the 12 target syllables (116msec syllable duration), for each of the three anchoring conditions, as well as the mean rate judgment for the 80- and 152-msec anchoring syllables, averaged over all three conditions. These data are displayed in Figure 3.

Consider first the anchoring stimuli. Averaged across conditions (slow, neutral, fast) and syllables (16- and 60-msec transition duration), the mean rate judgment was 8.41 for the 80 -msec anchoring syllables and 2.55 for the 152 -msec anchoring syllables. Thus, the fast and slow anchoring syllables were clearly perceived as having different rates. (We

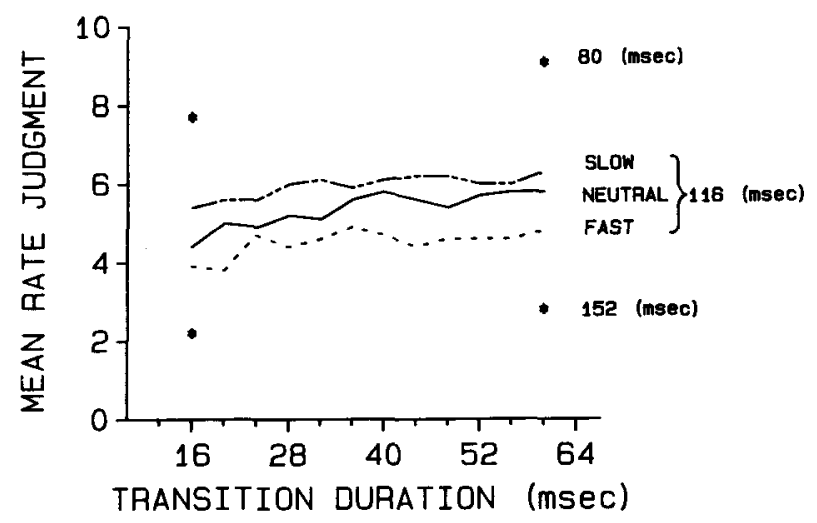

Figure 3. Mean rate judgment for syllables from the 116-msec target /ba-wa/ series in three anchoring conditions, and mean rate Judgment for the fast (80 msec) and slow (152 msec) anchoring syllables. Experiment 2. should point out that for both the 80 - and the 152 msec syllables, analyses of variance indicated that the judged rate of the anchoring syllables did not reliably differ across the three conditions, $p>.10$ in each case). Note that the large difference in rate judgments for the 80- and 152-msec syllables in this experiment contrasts with the relatively small difference in rate judgment between these stimuli in Experiment 1 (see Figure 2). This indicates that the subjects followed the instructions to judge rate in relation to the range of rates encountered in the experiment.

Consider next the rate judgments for the $116-\mathrm{msec}$ target syllables. It is apparent that we were successful in generating contrast effects: The rate judgments were highest in the slow anchoring condition and lowest in the fast anchoring condition, as predicted. To obtain a summary measure of rate judgment, we calculated, for each subject, the mean rate judgment across all 12 stimuli in the series, for each condition. These values, pooled across subjects, are 5.96, 5.37, and 4.46, for the slow, neutral, and fast conditions, respectively. Thus, the mean difference in rate judgment between the slow and neutral condition is .59, and between the neutral and fast condition it is .91. To determine the reliability of the anchoring effect, the individual scores for the 116 -msec series (averaged over the 12 stimuli in the series), in the three conditions, were entered into a one-way repeated measures analysis of variance. We obtained a highly significant effect of condition $[F(2,26)=79.66, p<$ $.001]$. Moreover, individual comparisons revealed that the mean rate judgments for both the slow and the fast conditions were reliably different from those of the neutral condition ( $p<.01$ in each case). Thus, although the contrast effects were small, they were highly reliable.

Given reliable differences in subjective rate among the three conditions, the critical question now is whether there were also reliable differences in boundary location. The identification functions for the 116msec series in the three conditions are displayed in Figure 4. (The anchoring stimuli were correctly identified on virtually all of the trials). The mean boundary values (calculated as in Experiment 1) for the three conditions are 34.81 (slow), 33.60 (neutral), and 35.01 (fast). Note that these values are not ordered in accord with the subjective rate hypothesis. And, a one-way repeated measures analysis of variance on the individual boundary scores indicated that the boundaries were not reliably different in the three conditions $[F(2,26)=2.17, p>.10]$. Thus, the modifications in subjective rate were not accompanied by reliable or even orderly differences in the /ba-wa/ boundary locations.

The results of this experiment are clearly in accord with the physical rate hypothesis, which claims that listeners adjust for changes in physical rate of speech, 


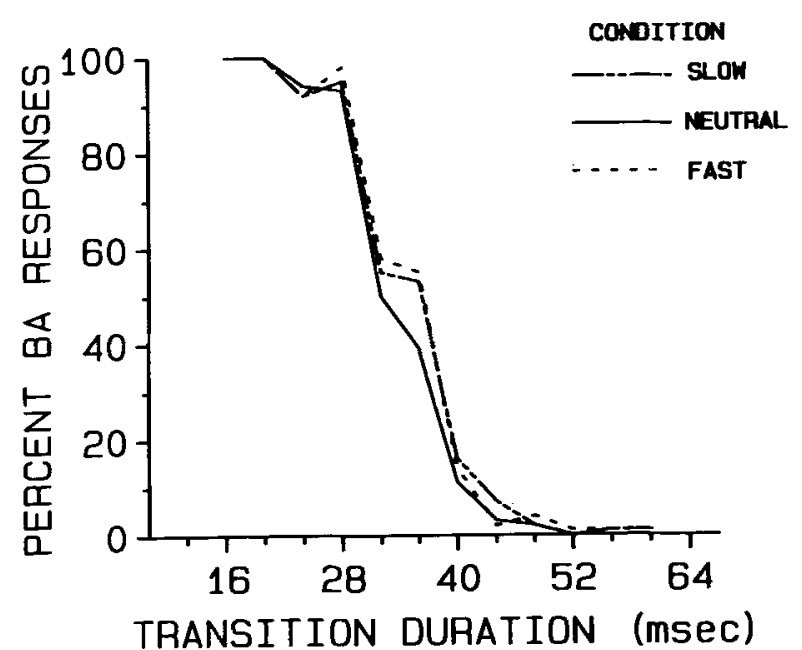

Figure 4. Percentage of /ba/ responses as a function of transition duration, for the 116-msec target /ba-wa/ series in three anchoring conditions. Experiment 2.

rather than for changes in subjective rate. However, before we take the results as strong evidence in support of this hypothesis, two possible alternative explanations of the findings must be considered. The first posits that the change in the rate judgments as a function of anchoring condition was due not to a change in subjective rate, as we have been assuming, but instead to an attempt by the subjects to use the rating categories equally often (cf. Parducci, 1975). That is, it was the trend toward equalizing use of response categories that produced more high-rating responses in the condition with many slow anchoring stimuli, which received low ratings, and more lowrating responses in the condition with many fast anchoring stimuli, which received high ratings. We should point out that the issue of the locus of the anchoring effect, and of context effects in general, has undergone considerable debate both in the speech perception literature and in the larger psychophysical literature. Although a complete resolution of this issue is not at hand, there are experiments that support the view that changes in context conditions produce alterations in subjective experience, and not simply alterations in overt response tendencies (e.g., Helson \& Kozaki, 1968; Sawusch \& Nusbaum, 1979).

Consider, in particular, the Sawusch and Nusbaum (1979) experiment. These investigators used an anchoring procedure nearly identical to the one we used to assess the effects of anchoring on the identification of members of an /i-I/ vowel series. As expected on the basis of previous work, increasing the frequency of occurrence of the endpoint / $i$ / stimulus produced an increase in the number of $/ I /$ responses, and, conversely, increasing the frequency of occurrence of the endpoint $/ \mathbf{I} /$ stimulus produced an increase in the number of $/ i$ / responses. Of particular relevance to the issue at hand is that the same mag- nitude of effect occurred even when the instructions to the subjects explicitly included the information that the tape would contain additional / $\mathrm{i} /$ or / $/$ / stimuli, so that they would expect to use the response categories an unequal number of times. As Sawusch and Nusbaum (1979) argued, these findings would seem to rule out an account of anchoring effects based on a strategy to equalize use of the response categories. Since our anchoring procedure was nearly identical to theirs, it is reasonable to assume that our effects were also not due to a shift in overt labeling but, instead, to an alteration in subjective experience.

A second alternative explanation concerns the magnitude of the context effects we obtained. Although the anchoring procedure was successful in producing reliable differences in the subjective rate of the target syllables, the effect was small. Consequently, it is possible that the changes in subjective rate were not large enough to produce reliable boundary shifts, and not that subjective rate is irrelevant. One way to rule out this possibility would be to demonstrate that changes in subjective rate of the magnitude we obtained through contrast, if obtained instead by changes in physical rate (syllable duration), are sufficient to yield significant boundary shifts. Experiment 3 was designed for this purpose.

\section{EXPERIMENT 3}

The aim of Experiment 3 was to determine whether changes in physical rate (syllable duration) that produce changes in subjective rate of the same magnitude as those produced through the anchoring procedure in Experiment 2 yield reliable shifts in the /bawa/ category boundary. Our strategy was to obtain identification responses and rate judgments for syllables from a number of /ba-wa/ series that varied, in small increments of syllable duration, between the duration of our fast $(80 \mathrm{msec})$ and slow $(152 \mathrm{msec})$ anchoring stimuli from Experiment 2. With this design, the overall range in rate for both experiments is identical, so that it is appropriate to compare the magnitude of differences in rate judgments across experiments. In all, we tested seven /ba-wa/ series, whose overall syllable durations were $80,92,104$, $116,128,140$, and $152 \mathrm{msec}$. Of prime interest is the 116-msec series, which served as the target series in Experiment 2. Our intent was to assess which of the slower and which of the faster series differed in subjective rate from the 116 -msec series by the amount induced by the anchoring procedure in Experiment 2, and then assess whether the boundary locations for these series differed reliably from that for the 116msec series.

\section{Method}

Subjects. Ten college students who had not participated in either of the two previous experiments served as paid subjects in this experiment. None of the students reported any history of a speech or hearing disorder. 
Stimuli. The stimuli consisted of seven /ba-wa/ series; the 12 syllables in each series ranged in transition duration from 16 to 60 msec. The series differed from each other in overall syllable duration, which was $80,92,104,116,128,140$, and $152 \mathrm{msec}$. Three of the series, those with overall syllable durations of 80,116 , and $152 \mathrm{msec}$, were from the stimulus set used in Experiment 1. The other four series, those with overall syllable durations of 92,104 , 128 , and $140 \mathrm{msec}$, were created by deleting the appropriate duration of the steady-state segment from the end of the syllable, in accord with the procedures used to create the original stimuli for Experiment 1.

Ten randomized test orders were recorded, each containing one instance of each of the 84 different stimuli (7 series $\times 12$ stimuli per series). The syllables were recorded with a constant onset-toonset interval of $4.4 \mathrm{sec}$.

Procedure. Each of the 10 subjects participated in one pretest session and four subsequent test sessions. During the pretest session, the listener was presented with three of the test orders, 6-8. Two subjects were excluded from the experiment, on the basis of the pretest, for failing to consistently identify the syllables as $/ \mathrm{ba} /$ and /wa/; they were replaced by additional subjects. During each of the four subsequent sessions, five test orders were presented, as follows: Sessions 2 and 4, orders 1-5; Sessions 3 and 5, orders 6-10.

The instructions to the subjects were the same as those of Experiment 2. Briefly, two judgments were required on every trial, first, an identification response ( $B$ or $W$ ) and, second, a rate judgment (1-10). And, as in Experiment 2, the subjects were told to judge the rate of each syllable in relation to the range of rates encountered in the experiment. In all, each subject provided 20 identification responses and 20 rate judgments for each stimulus.

As in the previous experiments, the stimuli were presented to subjects binaurally over matched TDH-39 earphones, and the levels were set so that the endpoint /ba/ (16-msec transition duration) from the 296 -msec series had a peak level of $78 \mathrm{~dB}$ SPL.

\section{Results and Discussion}

The rate-judgment data, displayed in Figure 5, clearly indicate that successive increments in overall syllable duration of $12 \mathrm{msec}$ yielded systematic differences in subjective rate. The overall mean rate judgment for each of the seven series, averaged across the 12 members of the series and the 10 subjects, are as follows: $8.27(80), 7.46(92), 6.28(104), 5.35$ (116), 4.45 (128), $3.82(140)$, and 3.41 (152).

Focusing first on the 116 -msec series, we note that the mean rate judgment of 5.35 found in the current experiment is very close to the mean judgment of 5.37 that was obtained for this series in the neutral anchoring condition of Experiment 2. Given this close correspondence, we can ask next which of the series in this experiment yielded values closest to those produced by the slow and fast anchoring conditions in Experiment 2, namely, 5.96 and 4.46, respectively. An examination of the mean scores reported above indicates that it is the 104- and 128msec series, with mean rate judgments of 6.28 and 4.45 , respectively. Note that the actual difference in rate judgment between the slow and neutral conditions in Experiment 2 was .59, whereas the difference between the 104- and 116-msec series in this experiment was .93. And the difference in mean rate judgment in Experiment 2 between the neutral and fast conditions was .91 ; in the current experiment, the difference between the 116- and 128-msec series was

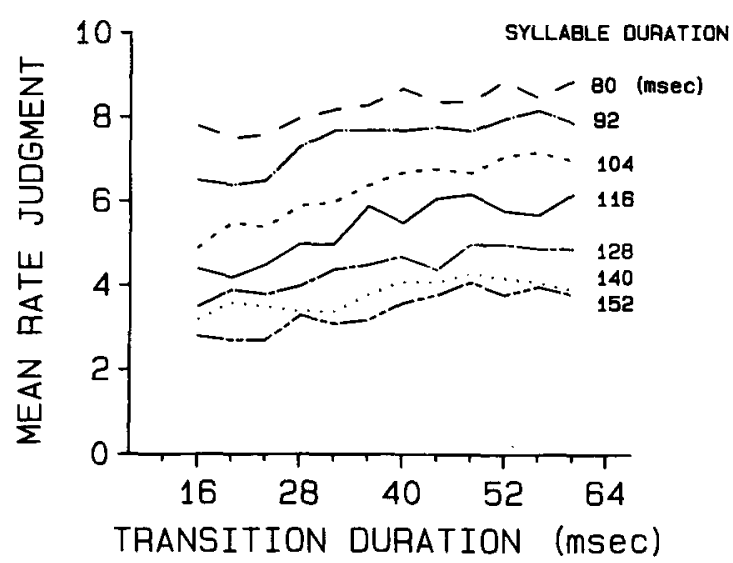

Figure 5. Mean rate judgment for syllables from seven /ba-wa/ series varying in overall syllable duration from 80 to 152 msec. Experiment 3.

.90 . Thus, we were reasonably successful in producing shifts in subjective rate of equal magnitude in the two experiments. Of course, it is important to know that the differences in mean rate judgment in this experiment are reliable, as they were in Experiment 2. A one-way repeated measures analysis of variance on the individual rate-judgment data (averaged across stimuli within each of the seven series), yielded a highly reliable effect of condition $[F(6,54)=94.55$, $\mathrm{p}<.001]$. More important, individual comparisons revealed that the difference in rate judgment between the 104- and 116-msec series and between the 116and 128 -msec series were reliable $(p<.01$ in each case).

Given the reliable differences in subjective rate for these series, of importance is whether they also differ in /ba-wa/ category boundary location. The identification data for all seven series, shown in Figure 6, reveal systematic changes in identification responses with changes in physical rate, as we would expect. The overall mean boundary locations (in millisecond

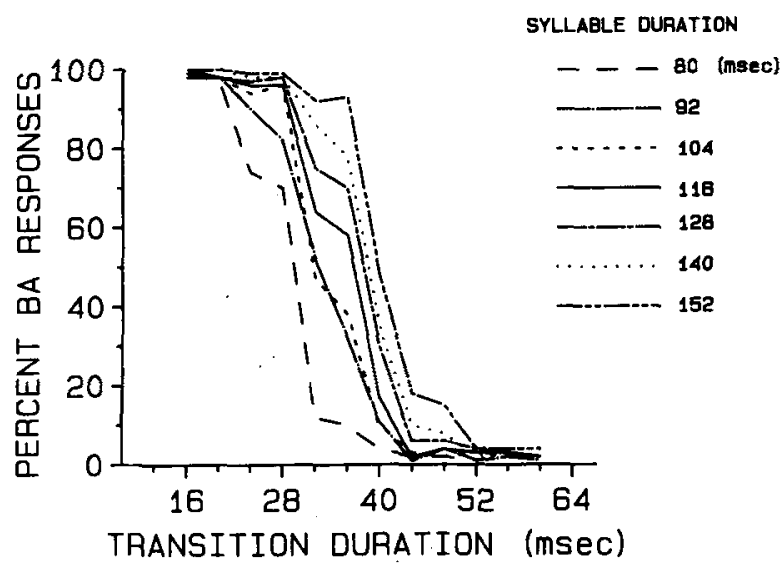

Figure 6. Percentage of $/ \mathrm{ba} /$ responses as a function of transition duration for seven /ba-wa/ series varying in overall syllable duration from 80 to 152 msec. Experiment 3. 


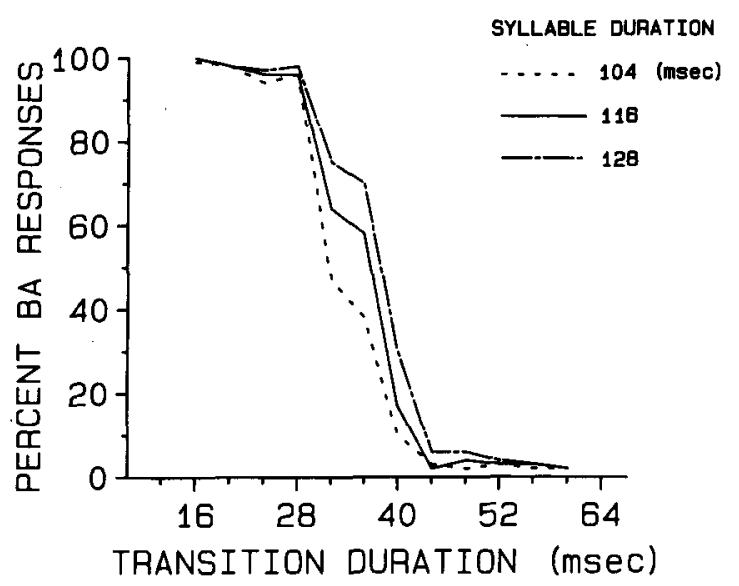

Figure 7. Percentage of /ba/ responses as a function of transition duration for / $b a-w a /$ series with overall syllable durations of 104, 116, and 128 msec. Experiment 3.

transition duration) for the seven series, calculated as previously described and averaged across subjects, are as follows: $29.37(80), 33.26(92), 34.08$ (104), 35.85 (116), $37.38(128), 38.59(140)$, and $40.48(152)$. Note that the value for the 116 -msec series of 35.85 agrees well with the value of 36.94 obtained for this series in Experiment 1 and the value of 34.47 obtained in Experiment 2 (averaged across conditions). The individual boundary locations for all seven series were entered into a one-way repeated measures analysis of variance. A highly reliable overall effect of condition was obtained $[F(6,54)=76.40, p<.001]$. Of critical importance for our purpose is whether the 104-, 116-, and 128-msec series, which are shown separately in Figure 7, differ reliably from each other in boundary location. Individual comparisons revealed that both the 104-msec series and the 128msec series differed reliably from the $116-\mathrm{msec}$ series ( $p<.01$ and $p<.02$, respectively). Comparing across the two experiments, we see that a difference in subjective rate of .90 (between the 116- and 128msec series) and a difference of .93 (between the 104and 116-msec series), which were induced by changes in physical rate, produced reliable boundary shifts, whereas a difference of 1.50 (between the fast and slow anchoring conditions of Experiment 2), which was produced without a change in physical rate, did not. In summary, then, the control experiment was successful in demonstrating that the small magnitude of the changes in subjective rate that we obtained through a context procedure is not, in itself, sufficient to explain the lack of a boundary shift in Experiment 2.

\section{GENERAL DISCUSSION}

The basis for the present research is the welldocumented finding that when listeners use durational properties of speech to identify phonetic seg- ments, they do so in a rate-dependent manner. Our purpose was to begin to elucidate the nature of this rate-dependent processing. In particular, we were interested in assessing two views of the listener's adjustment for speaking rate. On one view, the critical rate information to which the listener adjusts during phonetic perception is the subjective rate of the utterance. On the other, phonetic perception is not dependent on, or mediated by, subjective rate, but rather is influenced directly by the physical rate of the utterance. Taken together, the results of the three experiments we report provide clear evidence in support of the physical rate hypothesis.

Given that it is physical, rather than subjective, rate that influences phonetic perception, we can consider the manner in which this rate information might have its effect during speech processing. At least two alternatives can be distinguished. The first is that during phonetic perception the listener simultaneously extracts two types of information from the speech signal: information about the rate at which the speech was produced and information about segmental identity, provided in our case by syllable duration and transition duration, respectively. A decision regarding phonetic identity, e.g., /ba/ versus /wa/, is then made on the basis of the transitional information, in conjunction with the rate information. By this account, the information for rate is extrinsic to the information specifying the segmental unit (Summerfield, 1981), and it is used to normalize the segmental information. The current findings indicate that if this view is correct, then normalization is based on the physical rate of speech, rather than on its subjective rate, which can be influenced by such factors as context.

As has been pointed out by a number of investigators (e.g., Miller, 1981b; Nooteboom, 1981; Summerfield, 1981), there are problems with an extrinsic timing account. One of the most difficult is that acoustic properties specifying rate and those specifying segmental identity are not necessarily distinct. Consequently, the very same property may provide information both about the rate and about the identity of the phonetic segment that is to be normalized for rate. For example, syllable duration specifies not only rate, but also vowel quality, and the perception of vowel quality itself is influenced by rate (e.g., Verbrugge \& Shankweiler, 1977). This situation demands that, at the very least, decisions about rate and segmental identity are not made independently of one another, but rather that they are made in parallel, and interactively. Just how the listener might do so, however, remains a mystery.

An alternative account of how speaking rate might influence phonetic perception entails the assumption that the correct specification of the acoustic information underlying the segmental unit remains invariant under any transformation of the acoustic signal due 
to speaking rate. That is to say, timing is intrinsic to the information specifying the phonetic segment (Summerfield, 1981). Moreover, phonetic identification is based solely on this phonetically relevant information-there is no need to extract information from the signal about speaking rate because there is no need for a normalization process. The present findings are well accommodated by such a view. Since the acoustic properties of the syllable were unaltered by the anchoring procedure, the critical information for phonetic identity remained constant, and therefore there was no change in syllable identification. In essence, the change in subjective rate had no effect because during phonetic identification there is no normalization for rate, be it physical or subjective rate.

Although an intrinsic timing account provides, in principle, an elegant solution to the problems of selfnormalization inherent in the extrinsic timing account, it is not without its own difficulties. One problem is that it has been notoriously difficult to discover acoustic information underlying a phonetic segment that remains invariant under rate variation (or, for that matter, under variation due to speaker, phonetic context, and the like). For a flavor of the difficulty, consider the /ba-wa/ case investigated in the present experiments. Given the systematic relation between the transition duration at the /bawa/ boundary and syllable duration, it would not be difficult to provide a description of the critical information that incorporated syllable duration. However, we know from other experiments that this will not suffice. In particular, Miller and Liberman (1979, Experiment 3) have shown that the way in which one lengthens the syllable matters: Increasing syllable duration by extending the steady-state vowel segment produces a shift in the boundary toward longer transition durations (as in the present experiments), whereas increasing duration by adding formant transitions appropriate for a final stop consonant, so as to create /bad-wad/, produces a shift in the opposite direction (for a discussion of this effect, see Miller \& Liberman, 1979). Thus, the acousticphonetic structure of the syllable, as well as its duration, is important. To accommodate this finding within an intrinsic timing account, the description of the invariant critical acoustic information will necessarily be abstract.

A second problem with the intrinsic timing account has to do with its generality. Up to this point, we have limited our consideration to individual syllables. But there are numerous demonstrations that the effective rate information influencing phonetic identification is not confined to the syllable itself, although, interestingly, the rate of the target syllable does appear to have the greatest influence (e.g., Port \& Dalby, 1982; Summerfield, 1981). For example, changing the rate of a precursor sentence can influence the identification of a subsequent syllable (e.g., Port \& Dalby, 1982; Summerfield, 1981). And although this type of effect is often quite small, it is robust, even when the changes in rate are confined to syllables that are at least one syllable removed from the target item (Miller, Green, \& Schermer, 1982). The problem for the intrinsic timing account is that it is difficult to see how any definition of the critical phonetic information could plausibly incorporate information about the rate of a precursor phrase. In fact, within the framework of an intrinsic view, it has been argued that the critical acoustic information for a segment will be defined precisely over that stretch of speech that is a function of the articulatory events that produce it, and the rate of distant syllables clearly lies outside of this domain. Thus, even if an intrinsic timing account proves successful in explaining segmental constancy under rate variation of the syllable itself, an extrinsic timing account appears to be required to explain the sensitivity of the listener to the rate of more distant speech. In light of the possibility of such a two-process account of rate effects, it will be especially interesting to extend the current research to precursor effects. We have seen that it is the physical, rather than subjective, rate of the test syllable that influences segmental identity. The question is whether this is also true for the rate variation in a precursor phrase or whether, for the precursor information, it is the subjective rate that is critical. If it is, in fact, subjective rate, we will have clear evidence of two distinct processes operating to maintain segmental constancy under rate variation.

In summary, we have demonstrated, as have others before us, that when listeners use a durational property to specify phonetic identity, they take into account the rate at which the speech was produced. We have also provided evidence concerning the nature of this rate-dependent processing: Our data strongly suggest that when the listener adjusts for changes in speaking rate, it is the change in physical rate, and not subjective rate, that is critical.

\section{REFERENCE NOTES}

1. Miller, J. L., Grosjean, F., Gee, J. P., Lomanto, C. Manuscript in preparation, 1983.

2. Summerfield, A. Q. Aerodynamics versus mechanics in the control of voicing onset in consonant-vowel syllables. (In Speech perception No. 4). Belfast: Queen's University of Belfast, Department of Psychology, 1975.

\section{REFERENCES}

Eimas, P. D., \& Cornit, J. D. Selective adaptation of linguistic feature detectors. Cognitive Psychology, 1973, 4, 99-109.

Goldman-Ersler, F. Psycholinguistics: Experiments in spontaneous speech. New York: Academic Press, 1968.

Grosjean, F., \& Deschamps, A. Analyse contrastive des variables temporelles de l'anglais at due français: Vitesse de 
parole et variables composants, phénomènes d'hésitation. Phonetica, 1975, 31, 144-184.

HeLson, H. Adaptation level theory. New York: Harper \& Row, 1964.

Helson, H., \& Kozaki, A. Anchor effects using numerical estimates of simple dot patterns. Perception \& Psychophysics, 1968, 4, 163-164.

KıRK, R. E. Experimental design: Procedures for the behavioral sciences. Belmont, Calif: Wadsworth, 1968.

$K_{\text {LATT, D. }}$ H. Linguistic uses of segmental duration in English: Acoustic and perceptual evidence. Journal of the Acoustical Society of America, 1976, 59, 1208-1221.

Miller, J. L. Effects of speaking rate on segmental distinctions. In P. D. Eimas \& J. L. Miller (Eds.), Perspectives on the study of speech. Hillsdale, N.J: Erlbaum, 1981. (a)

Mrluer, J. L. Some effects of speaking rate on phonetic perception. Phonetica, 1981, 38, 159-180. (b)

MILLER, J. L., \& BAER, T. Some effects of speaking rate on the production of $/ \mathrm{b} /$ and $/ \mathrm{w} /$. Journal of the Acoustical Society of America, 1983, 73, 1751-1755.

Miller, J. L., Green, K., \& Schermer, T. On the distinction between prosodic and semantic factors in word identification. Journal of the Acoustical Society of America, 1982, 71, S95.

Miller, J. I., \& Liberman, A. M. Some effects of lateroccurring information on the perception of stop consonant and semivowel. Perception \& Psychophysics, 1979, 25, 457-465.

Noотевоом, S. G. Speech rate and segmental perception or the role of words in phoneme identification. In T. Myers, J. Laver, \& J. Anderson (Eds.), The cognitive representation of speech. Amsterdam: North Holland, 1981.

Parducci, A. Contextual effects: A range-frequency analysis. In E. C. Carterette \& M. P. Friedman (Eds.), Handbook of perception (Vol. 2). New York: Academic Press, 1975.

PonT, R. F. Linguistic timing factors in combination. Journal of the Acoustical Society of America, 1981, 69, 262-274.

PorT, R. F., \& Dalby, J. Consonant/vowel ratio as a cue for voicing in English. Perception \& Psychophysics, 1982, 32, 141-152.

Sawusch, J. R., \& Nusbaum, H. C. Contextual effects in vowel perception I: Anchor-induced contrast effects. Perception \& Psychophysics, 1979, 25, 292-302.
Simon, H. J., \& Studdert-Kennedy, M. Selective anchoring and adaptation of phonetic and nonphonetic continua. Journal of the Acoustical Society of America, 1978, 64, 1338-1357.

Summerfield, Q. On articulatory rate and perceptual constancy in phonetic perception. Journal of Experimental Psychology: Human Perception \& Performance, 1981, 7, 1074-1095.

Verbrugge, R. R., \& Shankweiler, D. Prosodic information for vowel identity. Journal of the Acoustical Society of America, 1977, 61, S39.

\section{NOTES}

1. In the Miller and Liberman (1979) paper, it is reported that the prevoiced segment of the syllables was $20 \mathrm{msec}$, rather than $16 \mathrm{msec}$. That is incorrect; for the original stimuli, as well as those created for this experiment, the prevoicing was $16 \mathrm{msec}$ in duration.

2. It is important to point out that the acoustic change produced by the increase in transition duration across the members of the /ba-wa/ series was actually quite complex. First, since the starting and terminating frequencies of the formants were kept constant, the increase in transition duration was necessarily accompanied by a decrease in the rate of transition. And second, since the overall amplitude of a syllable increased by a given amount over the course of the entire prevoicing and transition segments, as transition duration become longer, the amplitude onset became less abrupt. In essence, then, we altered a number of parameters that contribute to the abruptness of the syllable onset. For ease of explication, we refer to this complex change as one of transition duration.

3. All individual comparisons reported in this paper were made with the least significant difference test (Kirk, 1968), using twotailed alpha values.

4. A 12-step series, which omitted the stimulus with a 64-msec transition duration, was used instead of the original 13-step series, simply because this made it considerably easier to construct the test orders for the various anchoring conditions with the appropriate ratios of fast $/ \mathrm{medium} / \mathrm{slow}$ syllables.

(Manuscript received June 7, 1983; revision accepted for publication September $30,1983$. ) 